\title{
Impacts of a Mindful Eating Intervention on Healthy Food-related Behaviors and Mindful Eating Practices among Elementary School Children: A Pilot Study
}

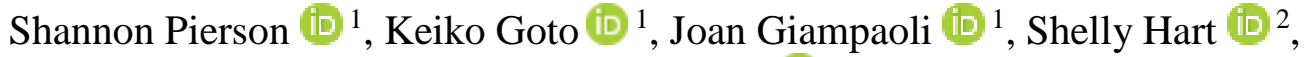 \\ and Alyson Wylie (iD ${ }^{3}$ \\ ${ }^{1}$ California State University, Chico, Department of Nutrition and Food Sciences \\ ${ }^{2}$ California State University, Chico, Department of Child Development \\ ${ }^{3}$ California State University, Chico, Center for Healthy Communities
}

\begin{abstract}
Background and Purpose: Mindful eating approaches encourage paying purposeful attention on purpose to body sensations, thoughts and emotions related to food and eating. The current study examined the impact of Foodie U, a mindful-eating intervention, among elementary school children and their families. Methods: A total of 178 third- through fifth grade children and their parents in rural northern California participated in Foodie U. The quasi-experimental intervention included two parent workshops, six monthly in-class mindful eating lessons and activities, and mindful eating activities to complete at home. Outcomes included food consumption, mindful-eating practices, emotional eating, and cue-elicited food craving among children. Results: T-tests and linear regression results revealed that students who received the intervention reported significantly less intense craving responses, especially among female and Hispanic students. The impact of the intervention on food craving was significant after controlling for gender and ethnicity. Fruit consumption significantly increased among intervention students. The mindful eating awareness score significantly increased among female intervention students. Conclusions: Foodie $\mathrm{U}$ had a positive impact on some food-related behaviors among elementary school-aged students. Further research is needed to examine the effectiveness of each element of the intervention.
\end{abstract}

(C) 2019 and CC-BY 4.0 licensed by the authors.

Keywords: Mindful eating; Children; Food craving

\section{Introduction}

Since 1980, the prevalence of obesity among young children has doubled, and the current rates of childhood overweight and obesity remain a troubling trend and major public health concern, especially among ethnic minorities (Ogden, Carroll, Kit, Flegal, 2014). According to 20152016 CDC NCHS data, among elementary school-aged children (6-11 years), $25.8 \%$ of Hispanic children are obese compared to $14.1 \%$ of non-Hispanic whites. Childhood overweight or obese has been associated with an increased risk of negative short-term health problems and tracking of obesity into adulthood (Reilly \& Kelly, 2011). Therefore, identifying factors affecting obesity in children and youth is imperative.
Research indicates that factors affecting unhealthy food behaviors leading to obesity are diverse, complex, and interrelated (Meule \& Kübler, 2014). Specifically, research supports that individuals with high Body Mass Index (BMI) experience more frequent food cravings, more episodes of binge eating, more eating in response to emotional situations, a greater disconnect from internal hunger and fullness cues, and are more sensitive to rewarding stimuli such as highly palatable foods than individuals with normal BMI (Meule \& Kübler, 2014). These unhealthy food behaviors can often be traced back to late childhood, just prior to the onset of puberty (Abbott et al., 1998). A study by Knez et al. (2006) indicates that disturbed eating attitudes and behaviors exist among elementary school children. Evidence from food-cue studies also 
indicates that children ages 6-12 experience more intense food cravings than do adolescents or young adults, and these years are associated with increased neural changes in regions of the brain linked to development of regulatory mechanisms (Silvers et al., 2014). Therefore, it is critical to develop and implement early intervention programs to teach children skills aimed at promoting a healthy relationship with food and obesity prevention prior to puberty.

\section{Socio-cultural and Environmental Factors}

There are various socio-cultural and environmental factors associated with unhealthy food behaviors among children (Wansink \& Sobal, 2007). Studies exploring the impacts of environmental food cues as they relate to intake patterns show that most Americans are very likely to rely on external cues to decide when to stop eating and are generally unaware when changes in their environment result in overconsumption (Wansink \& Sobal, 2007).

Environmental factors can affect consumption and satisfaction. For example, a smaller plate or serving size leads individuals to consume less food with equal satisfaction and fullness while using larger plates or portions makes them consume more. Food cue stimuli can also override biological signals to stop eating. Such stimuli can lead satiated individuals to seek and consume rewarding foods when they otherwise showed no motivation to seek food in the absence of the stimulus (Wansink \& Sobal, 2007). Consequently, these environmental food cues can easily contribute to "mindless eating" (Wansink, 2010). Children's unhealthy food behaviors are further exacerbated by food environment at home and school. Peer pressure among children and parents' feeding practices have been shown to influence children's food behaviors (Arredondo et al., 2006; Oliver \& Thelen, 1996). Specifically, among Hispanic students the loss of eating together as a family at home, has diminished due to acculturation (Batis, Hernandez-Barrera, Barquera, Rivera, \& Popkin, 2011). Consequently, healthier, traditional diet patterns have also diminished.

\section{Mindful Eating Approach}

In light of the many external factors influencing food behaviors among children, nutrition researchers and educators are interested in the mindful eating approach to change food behaviors. The mindful eating approach encourages paying purposeful attention to bodyrelated sensations and to thoughts and feelings about food (Alberts, Thewissen, \& Raes, 2012). This awareness and acceptance can strengthen attention to regulatory cues and decrease emotional eating due to negative emotions. Research suggests that mindful-eating interventions can effectively improve health outcomes for obese adults (O'Reilly, Cook, Spruijt-Metz, \& Black, 2014). Successful interventions reduced frequency and intensity of cravings, emotional eating, and binge-eating episodes; these results were associated with increases in mindfulness and mindful eating scores (O’Reilly et al., 2014).

There is limited information about the effectiveness of mindful-eating interventions among children especially in non-clinical settings. For example, food-cue research demonstrated evidence that children as young as six years old have been successfully taught to regulate craving (Silvers et al., 2014). However, it is not yet known whether general mindfuleating interventions in non-clinical settings can successfully alter children's response to external and internal food cues, emotional eating, and highly palatable food intake. Lastly, it is not yet known whether and how parents' involvement at home and mindful feeding practices with their children affect children's food behaviors. A recent study demonstrated that mindful food parenting with present-centered awareness predicted greater consumption of healthy foods and less consumption of unhealthy foods among children (Meers, 2013), providing the rationale for involving parents in a mindful eating intervention among children.

\section{Foodie U}

Foodie U: a mindful-eating intervention among children and families was a two-year seed grant project of the Agricultural and Food Research Initiative (AFRI) program funded through the United States Department of Agriculture 
(USDA). The primary goal of the intervention was to develop and pilot-test strategies based on mindful eating practices for fostering healthy food-related behaviors among elementary school children and their families, primarily consisting of Hispanic and non-Hispanic whites. The development and implementation of Foodie $U$ was described in an earlier article of this journal (Pierson et al., 2016). The intervention included 2 parent workshops about mindful eating, six 45to 60 minute monthly classroom lessons by nutrition educators, and six take-home activities. The current study aimed to evaluate the impact of the intervention on healthy food behaviors among elementary school children.

\section{Methods}

\section{Study Design}

The quasi-experimental study was designed to compare an intervention group with a control group. The intervention and control schools were from the same school district with similar geographic and socio-economic characteristics. Quantitative data was collected from student prepost surveys. All intervention activities and evaluation instruments were evaluated by experts including a child development specialist and were pilot tested prior to implementation.

\section{Participants}

Participants were recruited from one low-income, elementary school district in rural northern California. Total third through fifth grade enrollment at the intervention school was 248 students in ten classrooms. The control school also had 248 third-to-fifth graders enrolled at the beginning of the intervention. Parental consent to participate in the intervention was sent home and collected prior to administration of any preintervention surveys. Parental consent to participate was provided for 178 students at the intervention school and 127 students at the control school. Control students were provided with mindful eating handouts at the end of the intervention.

\section{Evaluation Instruments}

All instruments were based on a review of the literature, existing measures, input from nutrition experts, and pre-intervention focus group findings. Each instrument was examined by experts and was pilot-tested prior to data collection.

Student Food Consumption Survey (SFCS). The SFCS is a self-report, validated, ageappropriate survey (Fourney \& Bellow, 2008) about food consumption. This survey specifically measures the consumption of fruit, vegetables, and unhealthy energy-dense food. The highest possible score is 5.0. Reported values indicate the number of times the type of food was eaten yesterday.

Eating in Emotional Situations Questionnaire (EESQ). The EESQ is a validated 11-item survey used to measure eating in emotional situations due to emotion-driven or context-driven cues in low-income children in the $4^{\text {th }}$ grade and older (Rollins et al., 2011). It is appropriate for use among low-income Hispanic children. Children are asked to answer "yes" or "no" to indicate whether they eat in each of the eleven emotional situations. Scores are reported as a sum of the total number of "yes" responses.

Cue-elicited Food Craving (CFC). The CFC is an online tool adapted from Silvers et al. (2014). CFC is designed to quickly and affordably measure children's craving for food in response to external food cues using 12 food images. Participants viewed each food image for three seconds and use a 5-point scale to rate how much they want to eat the food they have just seen $(0=$ not at all; $1=$ a little bit; $2=$ somewhat; $3=$ quite a bit; 4=very much) (Silvers et al., 2014). Higher scores indicate more craving intensity.

Mindful Eating Questionnaire for Children (MEQ-C). Based on the Mindful Eating Questionnaire (MEQ), a validated measure designed to measure mindful eating skills among adults (Framson et al., 2009), the Mindful Eating Questionnaire for Children (MEQ-C) was developed and validated (Hart et al., 2018). MEQ-C has two subscales (awareness and mindless eating). The impact of the intervention on awareness and mindless eating was examined using MEQ-C. Average scores out of 4.0 were calculated. Higher scores signify greater frequency of awareness behaviors 
(1=never/rarely; $\quad 2=$ sometimes; $\quad 3=$ often; $4=$ usually/always). Since all eight items for "mindless eating" were originally reverse-coded, they were recoded ranging from 1 to 4 (1=never/rarely; $\quad 2=$ =sometimes; $3=$ often; 4=usually/always) so that higher scores signify more "mindless" eating behaviors.

\section{Intervention Procedures}

The research team held meetings with school principals, staff and teachers of the intervention and control schools to discuss the project content and gain approval for all project protocols. Different fliers about the project were developed for the intervention and control school parents. They were distributed to parents through school staff and teachers. The Human Subjects Research Committee of California State University, Chico granted ethical approval to conduct this study. Written parent/guardian consent was obtained for students to participate in the study.

The intervention consisted of six classroom lessons, related take-home activities and two parent workshops. Curriculum content was developed around Experiential Learning Theory (ELT), which posits that experience is transformed into knowledge through concrete experiences and abstract conceptualization (Kolb, Boyatzis, \& Mainemelis, 2001). The parent workshops aimed to enhance knowledge of mindful eating, as well as mindful cooking and eating skills among parents. The monthly topics included environmental factors affecting mindless eating, awareness of sensory characteristics of food, hunger and fullness, emotional eating, awareness and appreciation of food resources, and mindful eating through cultural and family traditions. Six take-home activities were given to students after each inclass lesson in order to foster mindful eating practices at home with parents. Bilingual (English/Spanish) take-home kits included an activity related to the topic of the month, a recipe, and/or non- perishable items used for the activity. The student pre-post surveys consisting of SFCS, MEQ-C, EESQ, and CFC were administered in the classroom using class sets of Chrome Book laptops. Student pre-intervention surveys took place prior to the first lesson to collect baseline data.

\begin{abstract}
Data Analysis
Quantitative survey data was analyzed with SPSSv22. Descriptive statistics were generated including frequencies and percentages to describe categorical variables and means and standard deviations to describe continuous or interval variables. Independent t-tests and Chi-square tests were used to assess differences in ethnic groups, grade levels, and gender between the intervention and control groups.
\end{abstract}

Of the students with consent to participate in the study, a total sample of 178 and 127 students were included in analyses from the intervention and control schools respectively. Nighty-six percent of intervention students completed the pre-survey and $84 \%$ completed the post-survey. Control group students pre- and post-survey participation was $84 \%$ and $89 \%$ respectively. Missing data were handled by using multiple imputation and pooled analyses to confirm results.

Paired t-tests were used to compare pre-to-post changes for mindful eating, emotional eating, and intensity of food craving among children. Independent t-tests were used to compare the mean pre-to-post changes in outcome scores between the intervention and control groups. These changes were also examined using linear regression. Ethnicity and gender were included in the models as covariates.

\section{Results}

Table 1 shows demographic characteristics of intervention and control students. There were significant differences in the distribution of student grade level and race. There were fewer $3^{\text {rd }}$ graders, more $5^{\text {th }}$ graders, and more Hispanic students at the intervention school.

Table 2 shows mean pre-to-post changes in the intervention and control groups. Grade level was not a significant factor associated with any outcome variables; however, gender and ethnicity were associated with craving and gender was associated with awareness (MEQ-C Factor 1), as shown in Table 2. 
Mean craving response decreased significantly among students who received the intervention $(\mathrm{p}=0.006)$. Furthermore, the independent $\mathrm{t}$-test revealed that the intervention group's pre-to-post change in craving response was significantly different from the control group $(\mathrm{p}<0.001)$. When comparing craving responses between genders at the intervention school, females showed a significant decrease $(\mathrm{p}=0.012)$ while males did not. Hispanic intervention students' craving significantly decreased $(\mathrm{p}=0.004)$ while nonHispanic students did not. Multivariate analysis revealed that there was a significant difference in pre-to-post craving response changes between intervention and control students after controlling for gender and ethnicity.

Table 1.

Participant Demographic Characteristics $(n=305)$

\begin{tabular}{lrr}
\hline $\begin{array}{l}\text { Student } \\
\text { Demographics }\end{array}$ & $\begin{array}{r}\text { Intervention } \\
\mathbf{n}(\%)\end{array}$ & $\begin{array}{r}\text { Control } \\
\mathbf{n}(\%)\end{array}$ \\
\hline Total n & & \\
& 178 & 127 \\
Grade Level & & \\
3rd & $49(27.5)$ & $51(40.2)$ \\
4th & $68(38.2)$ & $51(40.2)$ \\
5th & $61(34.3)$ & $25(19.7)$ \\
Gender & & \\
Female & $93(52.2)$ & $71(55.9)$ \\
Male & $85(47.8)$ & $56(44.1)$ \\
Race & & \\
White & $92(51.7)$ & $81(63.8)$ \\
Latino & $76(42.7)$ & $34(26.8)$ \\
Other & $10(5.6)$ & $12(9.5)$ \\
\hline
\end{tabular}

Among intervention students, there was a marginal pre-to-post change in MEQ-C Factor 1 (awareness) scores among intervention students $(\mathrm{p}=0.099)$. When comparing pre-to-post change in awareness between genders, female scores significantly increased $(\mathrm{p}=0.015)$ and there was no change among males $(\mathrm{p}=0.840)$. There was no significant pre-to-post change among control females $(\mathrm{p}=0.880)$. There were no significant preto-post changes in MEQ-C Factor 2 (mindless eating) scores. Fruit consumption among the intervention group increased $(\mathrm{p}<0.001)$. There was no significant pre-to-post change in fruit consumption between intervention and control groups. There were no other significant changes in consumption patterns, mindful eating, or emotional eating outcomes.

\section{Discussion}

The current study aimed to examine the impact of Foodie $U$, a mindful eating intervention among elementary school children and their families, on food-related behaviors and mindful eating practices among children. To our knowledge, this is one of the first studies that examined the effectiveness of a mindful-eating intervention among children. Results indicate that students who received the intervention reported significantly less intense craving responses $(\mathrm{p}=$ 0.006 ). Less intense craving responses were observed among female $(\mathrm{p}=0.012)$ and Hispanic $(p=0.004)$ intervention students. The impact of the intervention on craving remained significant after controlling for gender and ethnicity. Fruit consumption significantly increased among intervention students. There was a marginal preto-post change in awareness scores among intervention students. There was a significant pre-to-post change in awareness among female intervention students.

The current study demonstrated the effect of the intervention on significantly less food craving responses, an important outcome associated with overeating and obesity among children. Research shows that individuals who have a tendency to have more food craving and overeat may experience limited inhibitory control in the presence of palatable food cues because of an increased reward sensitivity (Meule \& Kübler, 2014). Research indicates that younger children (ages 6-12) experience more food cravings at a greater intensity than do adolescents or adults, likely because food cues stimulate increased activation within the region of the brain involved in reward-sensitivity among younger children (Colagiuri and Lovibond, 2015). However, children as young as six years have been taught to regulate craving with minimal training (Silvers et al., 2014). They found that participants reported less craving related to viewing images of appetizing unhealthy foods when purposefully regulating their responses by imagining the images as being farther away. Frequency and intensity of food cravings have been reduced 
among adults practicing mindful eating (O'Reilly et al., 2014). Research by Alberts et al. (2012) revealed that food cravings reduced significantly among female adults who participated in a mindfulness intervention. The results of the current study suggest that practicing mindful eating may have a similar regulatory effect on craving response among children. Future studies are needed to examine how mindful eating practices may reduce food reward sensitivity and food craving among children.

Table 2.

Pre-to-post Changes in Intervention Outcomes

\begin{tabular}{|c|c|c|c|c|c|c|c|c|}
\hline \multirow{2}{*}{ Children's } & \multicolumn{4}{|c|}{ Intervention group } & \multicolumn{4}{|c|}{ Control group } \\
\hline & & Pre & Post & & & Pre & Post & \\
\hline \multirow{4}{*}{$\begin{array}{l}\text { Children's } \\
\text { behaviors } \\
\text { MEQC Factor 1: } \\
\text { awareness }\end{array}$} & $\mathbf{N}$ & Mean (SE) & Mean (SE) & $\mathbf{p}$ & $\mathbf{N}$ & Mean (SE) & Mean (SE) & $\mathbf{p}$ \\
\hline & & 2.821 & 2.911 & & & 2.780 & 2.756 & \\
\hline & 178 & $(.048)$ & $(.050)$ & 0.099 & 127 & $(.055)$ & $(.065)$ & 0.764 \\
\hline & & 2.784 & 2.970 & & & 2.801 & 2.815 & \\
\hline \multirow[t]{2}{*}{ Females } & 93 & $(.066)$ & $(.070)$ & $0.015 *$ & 71 & $(.075)$ & $(.079)$ & 0.880 \\
\hline & & 2.861 & 2.845 & & & 2.752 & 2.681 & \\
\hline \multirow[t]{2}{*}{ Males } & 85 & $(.071)$ & $(.071)$ & 0.840 & 56 & $(.090)$ & $(.102)$ & 0.601 \\
\hline & & 2.847 & 2.987 & & & 2.752 & 2.752 & \\
\hline Hispanic & 76 & $(.068)$ & $(.063)$ & 0.080 & 34 & $(.092)$ & $(.111)$ & 1.000 \\
\hline non- & & 2.801 & 2.854 & & & 2.790 & 2.771 & \\
\hline Hispanic & 102 & $(.067)$ & $(.073)$ & 0.480 & 93 & $(.066)$ & $(.076)$ & 0.830 \\
\hline \multirow{3}{*}{$\begin{array}{l}\text { MEQC Factor 2: } \\
\text { mindless eating }\end{array}$} & & 2.894 & 2.929 & & & 2.941 & 2.884 & \\
\hline & 178 & (.039) & $(.040)$ & 0.375 & 127 & $(.050)$ & $(.047)$ & 0.338 \\
\hline & & 2.792 & 2.869 & & & 2.854 & 2.767 & \\
\hline \multirow[t]{2}{*}{ Females } & 93 & $(.048)$ & $(.050)$ & 0.165 & 71 & $(.057)$ & $(.057)$ & 0.236 \\
\hline & & 3.001 & 2.994 & & & 3.051 & 3.032 & \\
\hline \multirow[t]{2}{*}{ Males } & 85 & $(.061)$ & $(.064)$ & 0.868 & 56 & $(.076)$ & $(.074)$ & 0.817 \\
\hline & & 2.885 & 2.876 & & & 2.967 & 2.801 & \\
\hline Hispanic & 76 & $(.063)$ & $(.062)$ & 0.888 & 34 & $(.083)$ & $(.081)$ & 0.080 \\
\hline non- & & 2.900 & 2.968 & & & 2.935 & 2.917 & \\
\hline Hispanic & 102 & $(.049)$ & $(.051)$ & 0.198 & 93 & (.059) & $(.057)$ & 0.794 \\
\hline \multirow{3}{*}{$\begin{array}{l}\text { Food craving } \\
\text { response }\end{array}$} & & 3.727 & 3.506 & & & 2.901 & 3.446 & \\
\hline & 178 & $(.077)$ & $(.070)$ & $0.006 *$ & 127 & $(.123)$ & $(.080)$ & $<0.001$ \\
\hline & & 3.665 & 3.407 & & & 2.959 & 3.210 & \\
\hline \multirow[t]{2}{*}{ Females } & 93 & $(.110)$ & $(0.100)$ & $0.012 *$ & 71 & $(.105)$ & $(.157)$ & 0.127 \\
\hline & & 3.796 & 3.615 & & & 2.828 & 3.745 & \\
\hline \multirow[t]{2}{*}{ Males } & 85 & $(.106)$ & $(.091)$ & 0.116 & 56 & $(.171)$ & $(.111)$ & $<0.001$ \\
\hline & & 3.773 & 3.414 & & & 2.759 & 3.243 & \\
\hline Hispanic & 76 & $(.117)$ & $(.108)$ & $0.004 *$ & 34 & $(.224)$ & $(.127)$ & 0.023 \\
\hline non- & & 3.695 & 3.574 & & & 2.963 & 3.528 & \\
\hline Hispanic & 102 & $(.101)$ & $(.087)$ & 0.213 & 93 & $(.133)$ & (.099) & $<0.001$ \\
\hline \multicolumn{9}{|l|}{ Food consumption } \\
\hline \multirow{3}{*}{$\begin{array}{l}\text { Fries and } \\
\text { chips }\end{array}$} & & 0.55 & 0.60 & & & 0.48 & .49 & \\
\hline & 178 & $(.062)$ & $(.070)$ & 0.537 & 127 & $(.071)$ & $(.068)$ & 0.890 \\
\hline & & 1.59 & 1.75 & & & 1.44 & 1.49 & \\
\hline \multirow[t]{2}{*}{ Vegetables } & 178 & $(.114)$ & $(.128)$ & 0.307 & 127 & $(.128)$ & $(.156)$ & 0.799 \\
\hline & & 1.59 & 2.11 & & & 1.51 & 1.88 & \\
\hline \multirow[t]{2}{*}{ Fruits } & 178 & $(.114)$ & $(.133)$ & $<0.001 *$ & 127 & $(.122)$ & $(.16)$ & 0.059 \\
\hline & & 0.73 & 0.63 & & & .50 & .63 & \\
\hline \multirow[t]{2}{*}{ Soda } & 178 & $(.065)$ & $(.064)$ & 0.262 & 127 & $(.065)$ & $(.077)$ & 0.203 \\
\hline & & 0.54 & 0.47 & & & .45 & .56 & \\
\hline \multirow{3}{*}{\begin{tabular}{l}
\multicolumn{1}{c}{ Sweets } \\
Eating in emotional \\
situations
\end{tabular}} & 178 & $(.058)$ & $(.054)$ & 0.354 & 127 & $(.065)$ & $(.067)$ & 0.158 \\
\hline & & & 2.976 & & & 3.268 & 3.000 & \\
\hline & 178 & $3.046(.180)$ & $(.177)$ & 0.714 & 127 & $(.217)$ & $(.210)$ & 0.221 \\
\hline
\end{tabular}


The current study demonstrated the effect of the intervention on significantly less food craving responses, an important outcome associated with overeating and obesity among children. Research shows that individuals who have a tendency to have more food craving and overeat may experience limited inhibitory control in the presence of palatable food cues because of an increased reward sensitivity (Meule \& Kübler, 2014). Research indicates that younger children (ages 6-12) experience more food cravings at a greater intensity than do adolescents or adults, likely because food cues stimulate increased activation within the region of the brain involved in reward-sensitivity among younger children (Colagiuri and Lovibond, 2015). However, children as young as six years have been taught to regulate craving with minimal training (Silvers et al., 2014). They found that participants reported less craving related to viewing images of appetizing unhealthy foods when purposefully regulating their responses by imagining the images as being farther away. Frequency and intensity of food cravings have been reduced among adults practicing mindful eating (O'Reilly et al., 2014). Research by Alberts et al. (2012) revealed that food cravings reduced significantly among female adults who participated in a mindfulness intervention. The results of the current study suggest that practicing mindful eating may have a similar regulatory effect on craving response among children. Future studies are needed to examine how mindful eating practices may reduce food reward sensitivity and food craving among children.

The current study showed some positive change in the first MEQ-C factor, mindful eating awareness. During one of the class lessons, intervention students explored all sensory attributes of a food and discussed the value of taking time to enjoy all that a food has to offer (Pierson et al., 2016). Another class lesson focused on the awareness of the feeling and meaning of hunger and fullness cues. Thus, those class activities may have significantly contributed to an increase in awareness among intervention students. Additionally, the findings of this study suggest that females experienced a greater change in both craving and awareness than males. A similar pattern in craving response was observed for Hispanic students (more change) than non-Hispanic students. Research has shown gender differences in response to a school-based mindfulness intervention. A recent study by Kang et al. (2018) indicates that female six-graders benefited more from a school-based mindful intervention compared to male sixgraders. As for ethnicity differences in response to our intervention, it is possible that bilingual (English and Spanish) take-home activity kits that included an activity card, a recipe, and/or nonperishable items were effective in promoting mindful eating practices among Hispanic families. Furthermore, some traditional food behaviors central to the Hispanic culture, such as eating together and cultural food consumption, were promoted in one of the lessons (Pierson et al., 2016). Many Hispanic families are losing such traditional behaviors (Batis et al., 2011), and this intervention may have helped them increase the awareness of such behaviors. Further research is needed to find out why female and Hispanic students responded to this intervention more than male and non-Hispanic students.

While there was a positive change in awareness regarding mindful eating, the intervention did not significantly improve mindless eating among children. The eating environment is filled with various external cues that impact eating choices such as the mealtime atmosphere, time of day, distractions, social interactions, and the ease or difficulty of accessing the food (Wansink \& Sobal, 2007). There are also environmental factors related directly to food, including the package size of a product, the portion size served, the variety of foods served, dinnerware size, shape and arrangement of serving containers, and convenient access to large amounts of food, all of which may influence mindless eating. Although one of the class lessons and a take-home activity addressed some environmental factors associated with mindless eating (Pierson et al., 2016), these activities may not have been sufficient for reducing mindless eating among children. Future interventions may need to focus on more awareness of various environmental factors and various activities that prevent mindless eating, such as portion size control. 
Additionally, the intervention did not significantly change emotional eating or food consumption except fruit consumption among children. According to Knez et al. (2006), elementary-school students may experience disturbed eating behavior as a result of physical and emotional changes that they are not prepared for. One of the class lessons allowed students to address the differences between physical and emotional needs and the potential influence of emotions of eating behaviors (Pierson et al., 2016). The lesson also included an activity to increase non-judgmental awareness of emotions. It is possible that the intervention was effective in increasing awareness of their own eating patterns associated with emotions, but it will take more time to observe actual eating changes.

There are several limitations in this study. While having a control group for comparison strengthens study design, the use of a small sample focused on Hispanic and other lowincome families and the lack of randomization limits generalization. Additionally, tools for evaluation were limited to self-reported student surveys. Finally, the current intervention may not have been long enough for some behavioral changes among children. The monthly classroom lessons and take-home activities lasted only six months. A longer intervention may have led to other behavioral changes in addition to food craving response, mindful eating awareness, and fruit consumption.

\section{Conclusion}

The long-term goal of this project was to improve the health of children and their families via healthy eating behaviors, and the current project establishes a foundation for this long-term goal. Findings from this study, such as a reduction in craving response and improvements in awareness of internal cues when eating, indicate that a controlled intervention to improve unhealthy food behavior for children is warranted. Further research is needed to examine the effectiveness of each element of the intervention. Additionally, information about socio-cultural factors associated with mindful eating practices and other outcomes from this current study will be useful in developing an effective intervention in the future. Finally, further testing and refinement of intervention materials and research tools are needed to improve the reliability of the results prior to conducting a controlled intervention.

\section{Acknowledgements}

This study was funded by a grant from the USDA National Institute of Food and Agriculture -Agriculture and Food Research Initiative (AFRI) program (Grant/Award \#: 2015-69001-23238). The authors would like to thank the research assistants for their support.

\section{References}

Abbott, D., de Zwaan, M., Mussell, M., Raymond, N., Seim, H., \& Crow, S. (1998). Onset of binge eating and dieting in overweight women: Implications for etiology, associated features and treatment. Journal of Psychosomatic Research, 44, 367-74.

Alberts, H. J. E. M., Thewissen, R., \& Raes, L. (2012). Dealing with problematic eating behaviour. The effects of a mindfulness-based intervention on eating behaviour, food cravings, dichotomous thinking and body image concern. Appetite, 58(3), 847-851. https://doi.org/10.1016/j.appet.2012.01.009

Arredondo, E. M., Elder, J. P., Ayala, G. X., Campbell, N., Baquero, B., \& Duerksen, S. (2006). Is parenting style related to children's healthy eating and physical activity in Latino families? Health Education Research, 21(6), 862-871. https://doi.org/10.1093/her/cyl110

Batis, C., Hernandez-Barrera, L., Barquera, S., Rivera, J. A., \& Popkin, B. M. (2011). Food acculturation drives dietary differences among Mexicans, Mexican Americans, and non-Hispanic Whites. Journal of Nutrition, 141(10), 1898-1906.

Colagiuri, B., \& Lovibond, P.F. (2015). How food cues can enhance and inhibit motivation to obtain and consume food. Appetite, 84, 79-87. 
Fourney, A., \& Bellow, A. (2008). 2005-2006 impact evaluation final report for Harvest of the Month.

Framson, C., Kristal, A., Schenk, J., Littman, A., Zeliadt, S., \& Beitez, D. (2009). Development and validation of the Mindful Eating Questionnaire. Journal of the American Dietetic Association, 109(8), 1439-1444. https://doi.org/10.1016/j.biotechadv.2011.08.021.Secreted

Hart, S.R., Pierson, S., Goto, K., \& Giampaoli, J.(2018). Development and initial validation evidence for a mindful eating questionnaire for children. Appetite, 129, 178-185.

Hales, C.M., Carroll, M.D., Fryar, C.D., \& Ogden, C.L.(2017). Prevalence of obesity among adults and youth: United States, 2015-2016. NCHS data brief, no 288. Hyattsville, MD: National

Center for Health Statistics.

Kang, Y., Rahrig, H., Eichel, K., Niles, H.F., Rocha, T., Lepp, N.E., Gold, J., \& Britton, W.B. (2018). Gender differences in response to a school-based mindfulness training intervention for early adolescents. Journal of School Psychology, 68, 163-176.

Knez, R., Munjas, R., Petrovecki, M., Paucić-Kirincić, E., \& Persić, M.(2006). Disordered eating attitudes among elementary school population. Journal of Adolescent Health, 38(5), 628-30.

Kolb, D. A., Boyatzis, R. E., \& Mainemelis, C. (2001). Experiential learning theory: Previous research and new directions. In Perspectives on thinking, learning, and cognitive styles (pp. 227-247).

Meers, M. R. (2013). The assessment of mindful food parenting and its relation to parental feeding practices and child food intake, PhD dissertation. Bowling Green State University, 2013.

Meule, A., \& Kübler, A. (2014). Double trouble. Trait food craving and impulsivity interactively predict food-cue affected behavioral inhibition. Appetite, 79, 174-182. https://doi.org/10.1016/j.appet.2014.04.014

O’Reilly, G. A., Cook, L., Spruijt-Metz, D., \& Black, D. S. (2014). Mindfulness-based interventions for obesity-related eating behaviours: A literature review. Obesity Reviews, 15(6), 453-461. https://doi.org/10.1111/obr.12156

Oliver, K. K., \& Thelen, M. H. (1996). Children's perceptions of peer influence on eating concerns. Behavior Therapy, 27(1), 25-39.

Pierson, S., Goto, K., Giampaoli, J., Wylie, A., Seipel, B., \& Buffardi, K. (2016). The Development of a mindful-eating intervention program among third through fifth grade elementary school children and their parents. Californian Journal of Health Promotion, 14(3), 70-76.

Rollins, B. Y., Riggs, N. R., Spruijt-Metz, D., McClain, A. D., Chou, C. P., \& Pentz, M. A. (2011). Psychometrics of the eating in emotional situations questionnaire (EESQ) among low-income Latino elementary-school children. Eating Behaviors, 12(2), 156-159.

Reilly, J.J., \& Kelly, J. (2011). Long-term impact of overweight and obesity in childhood and adolescence on morbidity and premature mortality in adulthood: Systematic review. International Journal of Obesisty, 35(7), 891-898.

Silvers, J. A., Insel, C., Powers, A., Franz, P., Weber, J., Mischel, W., ... Ochsner, K. N. (2014). Curbing craving: Behavioral and brain evidence that children regulate craving when instructed to do so but have higher baseline craving than adults. Psychological Science, 25(10), 1932-1942.

Wansink, B. (2010). From mindless eating to mindlessly eating better. Physiology and Behavior, 100(5), 454-463. https://doi.org/10.1016/j.physbeh.2010.05.003

Wansink, B., \& Sobal, J. (2007). Mindless eating: the 200 daily food decisions we overlook. Environment and Behavior, 39(1), 106-123. https://doi.org/10.1177/0013916506295573 
Corresponding Author Information

Keiko Goto (ORCID: 0000-0003-0620-3485)

Professor and Graduate Coordinator, Department of

Nutrition and Food Sciences

California State University, Chico

Chico, CA 95929

(530) 898-4022 Office

(530) 345-0668 Fax

kgoto@csuchico.edu 